\title{
WEAK SUBORDINATION OF MULTIVARIATE LÉVY PROCESSES
}

\author{
KEVIN W. LU
}

(Received 13 April 2019; first published online 9 July 2019)

2010 Mathematics subject classification: primary 60G51; secondary 60E07, 60E10, 62F10, 60J65, 60J75, $62 \mathrm{P} 05$.

Keywords and phrases: Brownian motion, gamma process, Lévy process, subordination, marked Poisson point process, Thorin measure, generalised gamma convolution, log return, Fourier inversion, method of moments, maximum likelihood estimation, self-decomposability, Bessel function.

Based on the idea of constructing a time-changed process, strong subordination is the operation that evaluates a multivariate Lévy process at a multivariate subordinator. This produces a Lévy process again when the subordinate has independent components or the subordinator has indistinguishable components; otherwise we prove that it does not in a wide range of cases. A new operation known as weak subordination is introduced, acting on multivariate Lévy processes and multivariate subordinators, to extend this idea in a way that always produces a Lévy process, even when the subordinate has dependent components. We show that weak subordination matches strong subordination in law in the previously mentioned cases where the latter produces a Lévy process. In addition, we give the characteristics of weak subordination and prove sample path properties, moment formulas and marginal component consistency. We also give distributional representations for weak subordination with ray subordinators, a superposition of independent subordinators, subordinators having independent components and subordinators having monotonic components.

The variance generalised gamma convolution class, formed by strongly subordinating Brownian motion with Thorin subordinators, is further extended using weak subordination. For these weak variance generalised gamma convolutions, we derive characteristics, including a formula for their Lévy measure in terms of that of a variance-gamma process, and prove sample path properties.

As an example of a weak variance generalised gamma convolution, we construct a weak subordination counterpart to the variance-alpha-gamma process of Semeraro.

Thesis submitted to the Australian National University in April 2018; degree approved on 16 October 2018; primary supervisor Boris Buchmann; co-supervisors Ross Maller and Alan Welsh.

(C) 2019 Australian Mathematical Publishing Association Inc. 
For these weak variance-alpha-gamma processes, we derive characteristics, show that they are a superposition of independent variance-gamma processes and compare three calibration methods: method of moments, maximum likelihood and digital moment estimation. As the density function is not explicitly known for maximum likelihood, we derive a Fourier invertibility condition. We show in simulations that maximum likelihood produces a better fit when this condition holds, while digital moment estimation is better when it does not. Also, weak variance-alpha-gamma processes exhibit a wider range of dependence structures and produce a significantly better fit than variance-alpha-gamma processes for the log returns of an S\&P500-FTSE100 data set, and digital moment estimation has the best fit in this situation.

Lastly, we study the self-decomposability of weak variance generalised gamma convolutions. Specifically, we prove that a driftless Brownian motion gives rise to a self-decomposable process and, when some technical conditions on the underlying Thorin measure are satisfied, that this is also necessary. Our conditions improve and generalise an earlier result of Grigelionis. These conditions are applied to a variety of weakly subordinated processes, including the weak variance-alpha-gamma process, and, in the previous fit, a likelihood ratio test fails to reject the self-decomposability of the log returns.

Some of the work in the thesis has been published in [1-3].

\section{Acknowledgements}

This research is supported by an Australian Government Research Training Program Scholarship and partially supported by a Chris Heyde Scholarship and the ARC grant DP160104737.

\section{References}

[1] B. Buchmann, K. W. Lu and D. B. Madan, 'Weak subordination of multivariate Lévy processes and variance generalised gamma convolutions', Bernoulli 25(1) (2018), 742-770.

[2] B. Buchmann, K. W. Lu and D. B. Madan, 'Calibration for weak variance-alpha-gamma processes', Methodol. Comput. Appl. Probab. (2018), to appear, doi:10.1007/s11009-018-9655-y.

[3] B. Buchmann, K. W. Lu and D. B. Madan, 'Self-decomposability of weak variance generalised gamma convolutions', Stoch. Process. Appl. (2019), to appear, doi:10.1016/j.spa.2019.02.012.

KEVIN W. LU,

Research School of Finance, Actuarial Studies and Statistics, Australian National University, Canberra, ACT 0200, Australia e-mail: kevin.lu@anu.edu.au 